\title{
Porcine Respiratory Cell and Tissue Coinfections and Superinfections with Porcine Reproductive and Respiratory Syndrome and Swine Influenza Viruses ${ }^{\dagger}$
}

\author{
Georges Saade 1, Caroline Hervet 1, Déborah Ménard 1, Patricia Renson 2, Céline Deblanc 2, \\ Juliette Bougon 2, Gaëlle Simon 2, Olivier Bourry 2, Nicolas Bertho ${ }^{1}$ and François Meurens 1,* \\ 1 BIOEPAR, INRAE, Oniris, 44307 Nantes, France; georges.saade@oniris-nantes.fr (G.S.); \\ caroline.hervet@oniris-nantes.fr (C.H.); Deborah.Menard@oniris-nantes.fr (D.M.); \\ nicolas.bertho@inrae.fr (N.B.) \\ 2 Laboratoire de Ploufragan-Plouzané-Niort, Unité de Virologie Immunologie Porcines ANSES, \\ Zoopôle Les Croix BP 53, 22440 Ploufragan, France; Patricia.RENSON@anses.fr (P.R.); \\ Celine.DEBLANC@anses.fr (C.D.); juliette.bougon@anses.fr (J.B.); Gaelle.Simon@anses.fr (G.S.); \\ Olivier.BOURRY@anses.fr (O.B.) \\ * Correspondence: francois.meurens@inrae.fr \\ + Presented at Viruses 2020-Novel Concepts in Virology, Barcelona, Spain, 5-7 February 2020. \\ Published: 19 June 2020
}

\begin{abstract}
Porcine reproductive and respiratory syndrome virus (PRRSV) and swine influenza type A virus (swIAV) are major contributors to the porcine respiratory disease complex that is still threatening porcine farming around the world. Understanding the interactions between these two viruses and the effect of their coinfection on the porcine immune response will lead to better preventive and therapeutic measures. In vivo studies of these coinfections showed contradictory results while in vitro studies showing slight interferences between the viruses were limited due to the fact that the two viruses did not share the same host cells. SwIAV mainly infects epithelial cells, while PRRSV only infects cells expressing CD163, such as alveolar macrophages. In an attempt to evaluate the effect of PRRSV infection on the replication of swIAV, the possible induction of trained immunity, and on the host antiviral response, we carried out several coinfections and superinfections using local viral strains. Our infection protocols were performed using a tracheal epithelial cell line and precision-cut lung slices (PCLS) combining different cell types mimicking infections in real conditions. The expression of viral and cellular transcripts involved in the recognition of the virus and in the antiviral response was assessed by quantitative PCR. Immunostaining was also carried out to monitor the virus distribution in the pulmonary tissue. Interestingly, without infecting epithelial cells, PRRSV was able to interfere with swIAV infection and inhibit the antiviral response of the hosting cell. The mechanisms of this interference are still unclear and need to be investigated to ultimately apply improved preventive and therapeutic approaches.
\end{abstract}

Keywords: coinfection

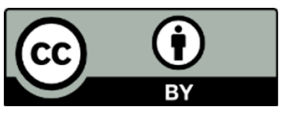

C 2020 by the authors. Licensee MDPI, Basel, Switzerland. This article is an open access article distributed under the terms and conditions of the Creative Commons Attribution (CC BY) license (http://creativecommons.org/licenses/by/4.0/). 